\section{6 OPEN ACCESS}

\title{
Endoscopic improvement of mucosal lesions in patients with moderate to severe ileocolonic Crohn's disease following treatment with certolizumab pegol
}

\author{
Xavier Hébuterne, ${ }^{1,2}$ Marc Lémann, ${ }^{2,3}$ Yoram Bouhnik, ${ }^{2,4}$ Olivier Dewit, ${ }^{5}$ \\ Jean-Louis Dupas, ${ }^{2,6}$ Michael Mross, ${ }^{7}$ Geert D'Haens, ${ }^{8}$ Krassimir Mitchev, ${ }^{9}$ \\ Étienne Ernault, ${ }^{9}$ Séverine Vermeire, ${ }^{10}$ Hedia Brixi-Benmansour, ${ }^{2,11}$ \\ Tom G Moreels, ${ }^{12}$ Jean-Yves Mary, ${ }^{2,13}$ Philippe Marteau, ${ }^{2,14}$ \\ Jean-Frédéric Colombel 2,15
}

- An additional table is published online only. To view this file please visit the journal online (http://dx.doi.org/10. 1136/gutjnl-2012-302262)

For numbered affiliations see end of article.

\section{Correspondence to} Professor Xavier Hébuterne, Department of Gastroenterology and Nutrition, Archet Hospital,

151, Route Saint Antoine de Ginestière, CHU de Nice, 06202 Nice Cedex 03, France; xavier.hebuterne@unice.fr

Marc Lémann died on 26 August 2010. We remember our esteemed colleague and recognise his contributions to this manuscript and to advancing the understanding and treatment of Crohn's disease.

Revised 27 February 2012 Accepted 29 February 2012 Published Online First 19 June 2012

\section{ABSTRACT}

Objective To evaluate the efficacy of certolizumab pegol (CZP) in improving endoscopic lesions in patients with active ileocolonic Crohn's disease (CD).

Methods This phase IIIB multicentre open-label clinical trial enrolled 89 adult patients with active endoscopic disease (ulceration in $\geq 2$ intestinal segments with a Crohn's Disease Endoscopic Index of Severity (CDEIS) score $\geq 8$ points). Patients received subcutaneous CZP $400 \mathrm{mg}$ at weeks 0,2 and 4 and every 4 weeks up to week 52. Endoscopic evaluations were performed at weeks 0,10 and 54 . The primary outcome was mean change in CDEIS score at week 10; secondary outcome measures included endoscopic response (decrease in CDEIS score $>5$ points), remission (CDEIS score $<6$ ), complete remission (CDEIS score $<3$ ) and mucosal healing (no ulcer) at weeks 10 and 54.

Results $\mathrm{In}$ the intention-to-treat population $(n=89)$ the mean \pm SD CDEIS score was $14.5 \pm 5.3$ at baseline; the mean decrease in CDEIS score at week 10 was 5.7 (95\% Cl 4.6 to 6.8, $\mathrm{p}<0.0001)$. Rates of endoscopic response, endoscopic remission, complete endoscopic remission and mucosal healing at week 10 were $54 \%$, $37 \%, 10 \%$ and $4 \%$, respectively. At week 54 the corresponding rates were $49 \%, 27 \%, 14 \%$ and $8 \%$, respectively. The safety profile was consistent with that of previous CZP trials.

Conclusions Following CZP treatment in patients with active $C D$, endoscopic lesions were improved as shown by the decrease in mean CDEIS score and by endoscopic response and remission rates. These benefits were achieved as early as week 10 and were generally maintained through week 54 .

\section{Clinical Trial Registration Number NCT00297648.}

\section{INTRODUCTION}

Crohn's disease (CD) is characterised by the presence of gut inflammation accompanied by areas of ulceration. ${ }^{1}$ Clinical response and remission have been and remain today the primary goals in the treatment of $\mathrm{CD}$. However, renewed interest in mucosal healing was raised by the finding that administration of infliximab, in addition to rapidly improving symptoms in patients with refractory

\section{Significance of this study}

What is already known on this subject?

- The efficacy of certolizumab pegol (CZP), a PEGylated anti-tumour necrosis factor, for induction and maintenance of response and remission has been demonstrated in adult patients with active Crohn's disease (CD).

- Improvement of endoscopic lesions and mucosal healing are emerging goals in the treatment of $C D$.

What are the new findings?

- Treatment with CZP $400 \mathrm{mg}$ every 4 weeks resulted in improvement of endoscopic lesions by week 10 in patients with moderate to severe ileocolonic CD.

How might it impact on clinical practice in the foreseeable future?

- The results of this study augment the available evidence that CZP $400 \mathrm{mg}$ every 4 weeks is effective in the treatment of CD.

luminal $\mathrm{CD}$, induced marked healing of ileocolonic lesions. ${ }^{2}$ In contrast to corticosteroids, ${ }^{3}$ evidence has since accumulated that treatment with immunosuppressors and/or biological agents is able to achieve long-term healing of the gut mucosa, which affects the clinical outcome of patients with active $\mathrm{CD}^{4}{ }^{5}$ Accumulating data suggest that mucosal healing in $\mathrm{CD}$ is associated with prolonged clinical remission and longer time to relapse, ${ }^{6-8}$ as well as with reductions in hospitalisations and operations. $^{8-10}$ Thus, mucosal healing is an increasingly important therapeutic goal in the treatment of patients with CD. ${ }^{411}$

The efficacy of certolizumab pegol (CZP), a PEGylated anti-tumour necrosis factor (TNF), for induction and maintenance of response and remission has been demonstrated in adult patients with active CD. ${ }^{12-14}$ The efficacy of CZP in producing mucosal healing has not yet been studied. The aim of this study was to evaluate the effects of CZP in
Scan to access more free content 
inducing and sustaining mucosal healing in patients with moderate to severe ileocolonic CD.

\section{PATIENTS AND METHODS}

The MUSIC (Endoscopic MUcoSal Improvement in Patients with Active Crohn's Disease Treated with CZP) trial was an open-label single-arm study over a period of 54 weeks with CZP in patients with moderate to severe ileocolonic CD and mucosal ulcers at colonoscopy. The primary objective of the study was to assess the effect of subcutaneous CZP $400 \mathrm{mg}$, administered at weeks 0, 2, 4 and 8, on endoscopic improvement of mucosal lesions in patients with active CD. The effect was assessed using the Crohn's Disease Endoscopic Index of Severity (CDEIS) score $^{15}$ at week 10 compared with baseline.

\section{Inclusion and exclusion criteria}

Adult men and women aged $\geq 18$ years with ileocolonic CD diagnosed for a minimum of 3 months and active disease (Crohn's Disease Activity Index (CDAI) $\geq 220$ and $<450$ scored over the 7 days prior to study drug initiation), requiring antiTNF treatment were eligible for the study. The presence of ulcerations was documented via endoscopy at screening, corresponding with a CDEIS score $\geq 8$ and at least two segments with endoscopic ulceration. Concomitant therapy with azathioprine, 6-mercaptopurine and methotrexate was allowed, provided that patients were receiving these medications for $\geq 8$ weeks prior to baseline and that doses had been stable for 4 weeks prior to baseline. Continued therapy with aminosalicylates or CD-related antibiotics was allowed, provided that patients had been on stable doses for $\geq 4$ weeks prior to baseline. Prednisone $30 \mathrm{mg} /$ day (or equivalent) or budesonide $9 \mathrm{mg} /$ day was permitted, provided patients were on stable doses for $\geq 2$ weeks prior to baseline. Patients with prior exposure to infliximab or any other anti-TNF agent (except CZP) were eligible, provided that they were not primary non-responders.

Patients with symptomatic intestinal strictures and fistulas, proctocolectomy or total colectomy or bowel resection within 4 weeks of initiation of study medication were excluded. Patients with total parenteral nutrition, short bowel syndrome, positive stool laboratory results for enteric pathogens or antibiotic therapy for non-CD-related infections within 3 weeks prior to screening were excluded. Additionally, there were numerous medical history exclusion criteria not associated with CD. Anti-TNF therapy was prohibited within 8 weeks of baseline and anti-TNF agents other than CZP could not be used during the study. The use of any investigational agent was prohibited within 8 weeks of baseline and during the study.

\section{Study design}

The study design is shown in figure 1 . After a screening period of 1 week, patients entered the 52 -week treatment period and a 2-week follow-up period. CZP $400 \mathrm{mg}$ subcutaneously was administered at weeks 0,2 and 4 (induction dose) and then every 4 weeks until week 52 . If neither a clinical response nor clinical remission were achieved by week 10, or if the clinical response was lost (clinician judgement) after week 10, the dose of CZP was escalated to $400 \mathrm{mg}$ every 2 weeks. Steroids were continued for the first 10 weeks at stable dosage; after 10 weeks steroid taper was permitted. Study visits occurred at 2 -week intervals from baseline to week 54. The CDAI was assessed for each patient at each visit.

Endoscopies were performed at baseline (week 0), after induction (week 10: primary endpoint) and at the end of the
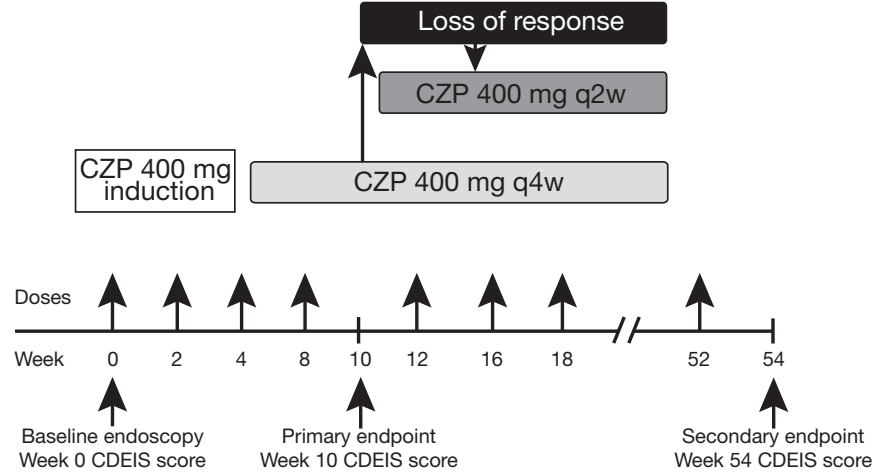

Figure 1 Study design. Loss of response was defined as both a Crohn's Disease Activity Index (CDAl) score $>150$ points and a minimum increase in CDAl of 70 points at two consecutive visits by week 10. CZP, certolizumab pegol; q2w, every 2 weeks; q4w, every 4 weeks; CDEIS, Crohn's Disease Endoscopic Index of Severity.

study (week 54). The endoscopist at the study site completed the CDEIS for each endoscopy. The CDEIS is a prospectively developed instrument that has been constructed to detect changes in endoscopic severity on the basis of characteristics of the ileocolonic mucosa. ${ }^{15}$ The extent of mucosal lesions was quantified on a visual analogue scale from 0 to 10 in five sections of the bowel: ileum, right colon, transverse colon, combined sigmoid and left colon, and rectum. In each segment the following items were recorded: surface of intestine affected by $\mathrm{CD}$, surface of ulcerated intestine, presence of superficial ulcers, presence of deep ulcers and stenosis. CDEIS scores ranged from 0 to 44, with greater scores indicating greater endoscopic severity. During endoscopy, biopsies were taken from inflamed mucosa and histological activity was calculated using the scoring system for histological abnormalities in CD mucosal biopsy specimens. ${ }^{16}$

Safety evaluations included adverse events/serious adverse events, vital signs, physical examinations and clinical laboratory tests (haematology, biochemistry and urinalysis). The frequency, severity, nature and duration of adverse events were assessed and recorded at each study visit.

\section{Central reading}

Investigators were asked to record the baseline, week 10 and week 54 endoscopies. After completion of the study, a central reading of endoscopies was performed. This process, which was not pre-specified in the MUSIC protocol, was undertaken as a means of assessing the accuracy of the local readings. Four expert readers blinded to the local readings and study time points scored the recorded endoscopies. The readers worked in pairs; each pair was randomly assigned to perform CDEIS scoring of all of the recorded endoscopies belonging to half of the patient population. For each pair, the average of the two CDEIS scores was the assigned CDEIS score. For the purpose of evaluating overall improvement in ulcerations and determination of mucosal healing, in the case of discrepancies between the two readers regarding the presence and severity of ulcers, the worse result was taken into account. Patients gave separate informed consent for the use of their recorded videotapes before any central reading was performed.

\section{Outcome measures}

The primary efficacy endpoint was mean change from baseline to week 10 in the CDEIS score. Main secondary endpoints were 
mean change in CDEIS score at week 54 relative to baseline; rates of endoscopic response, remission, complete remission, mucosal healing and proportion of patients with improvement of ulcerations at week 10 and week 54; maintenance of endoscopic effect at week 54 relative to week 10. Endoscopic response, remission and complete remission were defined in the Statistical Analysis Plan before any data analysis, according to the definitions of Mary et $a l^{17}$ as follows: endoscopic response as a decrease in the CDEIS score of $>5$, endoscopic remission as a CDEIS score $<6$, complete endoscopic remission as a CDEIS score $<3 .{ }^{17}$ Mucosal healing was defined as absence of ulceration. Improvement of ulcerations was defined by a change from deep ulcerations to superficial ulcerations or absence of ulcerations, or by a change from superficial ulcerations to absence of ulcerations between two visits. Maintenance of endoscopic effect was defined as the absence of change in mean CDEIS between week 10 and week 54 and, in addition, expressed through the proportions of patients with endoscopic improvement at week 54 among those with endoscopic improvement at week 10 and available endoscopy at week 54 .

Additional secondary endpoints were rates of clinical response and remission at weeks 10 and 54, mean histological scores at weeks 10 and 54 and mean $C$ reactive protein (CRP) concentration at each visit. Clinical remission was defined by a CDAI score of $\leq 150$ and clinical response by a decrease in CDAI score from baseline of $>100$.

Protocol-defined exploratory endpoints included association between CDEIS score and CDAI score or CRP levels at each time point. In line with the addition of the central reading after completion of the study, supplementary endpoints were agreement between CDEIS scores from paired experts of central readings and agreement between CDEIS score based on local readings and CDEIS score based on central readings at each time point.

\section{Statistical analysis}

All patients who were enrolled were included in the intentionto-treat (ITT) population. Regarding patients' characteristics at inclusion, continuous variables were evaluated using descriptive statistics (median, range, IOR; mean, SD); categorical variables were evaluated using frequency tables. The CDEIS score at each visit was expressed as mean $\pm \mathrm{SD}$. For the calculation of change in CDEIS score between two visits in the ITT population, a last observation carried forward imputation was performed when patients discontinued the study before second visit assessment or when the CDEIS score was not evaluated at that visit. The change in CDEIS score was expressed as mean (95\% CI). In addition, the change in CDEIS score was also evaluated in a subpopulation in which both CDEIS evaluations were available. For the assessment of endoscopic response, endoscopic remission, complete endoscopic remission, mucosal healing and improvement of ulcerations at a follow-up visit in the ITT population, patients with no CDEIS evaluation (either missing or not available due to discontinuation before the visit) were deemed treatment failures. To assess the maintenance effect from weeks 10 to 54, the mean (95\% CI) change in CDEIS score was evaluated in the subpopulation of patients with both evaluations, as well as the proportion of endoscopic response, endoscopic remission and complete endoscopic remission at week 54 among patients with endoscopic response, endoscopic remission and complete endoscopic remission at week 10. For the calculation of clinical response, patients who discontinued before the assessment of response were considered to be non-responders.
The Student $t$ test was used to assess significance for the primary efficacy variable. In the absence of a control group, results for all other efficacy variables were presented with CIs in order to test the null hypothesis of no variation in CDEIS score between two visits or of no response, remission, complete remission or mucosal healing at a follow-up visit. For the determination of sample size we used the data for the overall treatment group from D'Haens et $a l^{18}$ where the mean change in the CDEIS score from baseline was estimated to be -7.8 with a SD of 5.4. Setting type I error at 5\% (two-sided) and allowing for a 1.25 margin of error from baseline in the CDEIS score yielded a sample size of 72 patients. Allowing for a dropout rate of $15 \%$ resulted in a final sample size estimate of 85 patients.

Spearman's correlation coefficient was calculated to evaluate the association level between CDEIS score and CDAI score or CRP level at each time point. The intraclass correlation coefficient (ICC) was calculated to evaluate the reproducibility level of CDEIS scores among paired experts of central reading at each time point. The Bland and Altman method ${ }^{19}$ was used to describe the agreement between CDEIS scores based on local readings and CDEIS score based on central readings at each time point.

\section{RESULTS \\ Patients}

The disposition of the 118 patients who were screened is shown in figure 2. A total of 89 patients were enrolled and constituted the ITT population. Patient demographic data and baseline characteristics are shown in table 1 . A total of $88 \%(78 / 89)$ and $60 \%(53 / 89)$ of patients had an endoscopic evaluation at weeks 10 and 54 , respectively. Dose escalation after week 10 , performed according to the clinical judgement of the investigator, took place in 46 patients.

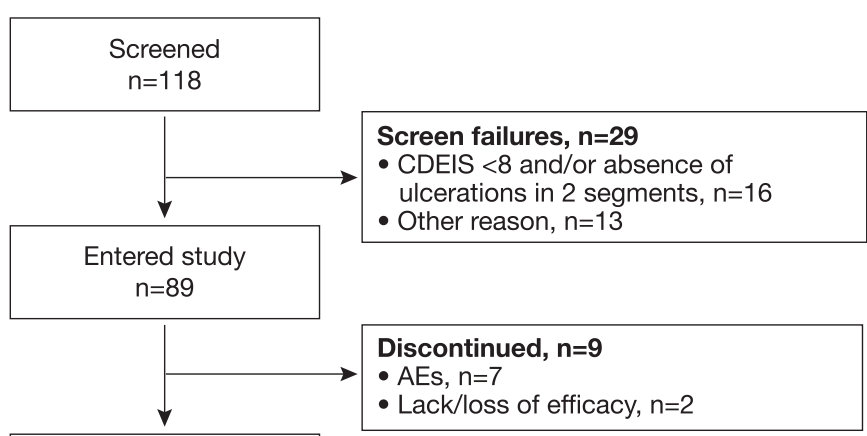

Completed treatment period to week $10, n=80$

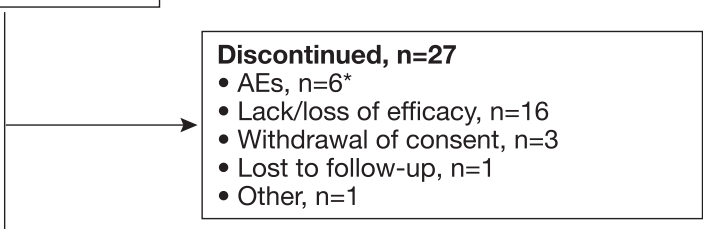

Completed treatment

period to week $54, \mathrm{n}=53$

Figure 2 Patient dispositions. * Includes one case each of anal fistula colonic stenosis, colon cancer, enterocutaneous fistula, gingivitis and pregnancy on oral contraceptive. $A E$, adverse event; CDEIS, Crohn's Disease Endoscopic Index of Severity. 
Table 1 Patient demographic data and baseline characteristics (intention-to-treat population)

\begin{tabular}{|c|c|}
\hline & $\begin{array}{l}\text { CZP } 400 \mathrm{mg} \\
(\mathrm{n}=89)\end{array}$ \\
\hline Age, mean $\pm S D$, years & $30.2 \pm 9.9$ \\
\hline $\mathrm{BMI}$, mean $\pm \mathrm{SD}, \mathrm{kg} / \mathrm{m}^{2}$ & $22.1 \pm 3.6$ \\
\hline Males, n (\%) & $30(34)$ \\
\hline \multicolumn{2}{|l|}{ CDEIS } \\
\hline Mean $\pm S D$ & $14.5 \pm 5.3$ \\
\hline Median & 13.3 \\
\hline IQR & $10.3-16.9$ \\
\hline \multicolumn{2}{|l|}{ CDAI } \\
\hline Mean $\pm S D$ & $297 \pm 75$ \\
\hline Median & 290 \\
\hline IQR & $236-342$ \\
\hline \multicolumn{2}{|l|}{ CRP, mg/l } \\
\hline Geometric mean (CV) & $19(1.4)$ \\
\hline Median & 29 \\
\hline IQR & $9-46$ \\
\hline \multicolumn{2}{|l|}{ Disease duration, years } \\
\hline Mean $\pm S D$ & $7.9 \pm 7.9$ \\
\hline Median & 6.5 \\
\hline \multicolumn{2}{|l|}{ Number of resections, $\mathrm{n}(\%)$} \\
\hline 0 & $71(80)$ \\
\hline 1 & $11(12)$ \\
\hline 2 & $5(6)$ \\
\hline 3 & $2(2)$ \\
\hline Prior anti-TNF therapy, $\mathrm{n}(\%)$ & $2(2)$ \\
\hline $\begin{array}{l}\text { At least one concomitant medication potentially } \\
\text { influencing } C D, \% \text { patients }\end{array}$ & 79 \\
\hline Immunosuppressants & 51 \\
\hline Corticosteroids & 42 \\
\hline 5-Aminosalicylic acid & 22 \\
\hline Antibiotics & 15 \\
\hline
\end{tabular}

BMI, body mass index; CD, Chron's disease; CDAl, Crohn's Disease Activity Index; CDEIS, Crohn's Disease Endoscopic Index of Severity; CRP, C reactive protein; CV, coefficient of variation; CZP, certolizumab pegol; TNF, tumour necrosis factor.

\section{Primary endpoint}

CDEIS scores observed at baseline, week 10 and week 54 in the ITT population and in the subpopulation with both evaluations are shown in table 2 . In the ITT population the mean change from baseline to week 10 in the CDEIS score was a decrease of 5.7 (95\% CI 4.6 to $6.8, \mathrm{p}<0.0001)$; among patients with endoscopic evaluation at week $10(\mathrm{n}=78)$ the mean decrease was $6.5(95 \%$ CI 5.3 to $7.6, \mathrm{p}<0.0001)$.

\section{Secondary endpoints}

In the ITT population $(\mathrm{n}=89)$, the mean decrease in CDEIS score from baseline to week 54 was 4.7 (95\% CI 3.5 to 6.0, p<0.0001); for patients with endoscopic evaluation at week $54(\mathrm{n}=53)$ the mean decrease was 5.5 (95\% CI 3.6 to $7.5, \mathrm{p}<0.0001)$.
Rates of endoscopic response, endoscopic remission, complete endoscopic remission and mucosal healing in the ITT population and in the subpopulation with endoscopic assessment at the corresponding visit are shown in table 2 . The improvement in ulcerations is shown in figure 3. A similar pattern of improvement was observed across all segments (data not shown). Overall, $47 \%$ of patients had a reduction in categorical severity of ulcers (ie, from deep to superficial or to absence, or from superficial to absence) between baseline and week 10 (ITT population with an endoscopic assessment at week 10, n=78) and $55 \%$ between baseline and week 54 (ITT population with an endoscopic assessment at week $54, n=53$ ). The frequency of specific sequences of ulcer severity across successive time points for this population is shown in table 1 in the online supplement.

With regard to maintenance of response, the mean change in CDEIS score between weeks 10 and 54 was $0.9(95 \% \mathrm{CI}-0.1$ to $2.0, \mathrm{p}=0.07)$ in the ITT population $(\mathrm{n}=89)$ and $1.8(95 \%$ CI 0.1 to $3.5, \mathrm{p}=0.04$ ) in the subpopulation with both evaluations $(n=52)$. Of the 37 patients in this subpopulation who were responders at week 10, 28 (76\%) maintained response at week 54. An additional six patients responded between weeks 10 and 54. Of the 24 patients in this subpopulation who were in remission at week 10, 11 (46\%) maintained remission at week 54. Of the seven patients in this subpopulation who were in complete remission at week 10 , five $(71 \%)$ maintained complete remission at week 54 .

In the ITT population $(n=89), 51 \%$ of patients had a clinical response and $46 \%$ attained clinical remission at week 10 ; 33\% of patients were clinical responders and $27 \%$ were in clinical remission at week 54 . The correlation coefficients between CDEIS and CDAI scores were 0.12 at week $0(n=85), 0.24$ at week $10(n=75)$ and 0.23 at week $54(n=40)$. In patients with endoscopic and histological assessment, the mean \pm SD histological score for the colon was 7.8 \pm 3.1 at week $0(n=87)$, $5.0 \pm 3.2$ at week $10(n=75)$ and $4.5 \pm 3.5$ at week $54(n=53)$. For the ileum the mean \pm SD histological score was $5.3 \pm 4.4$ at week $0(\mathrm{n}=79), 2.4 \pm 3.4$ at week $10(\mathrm{n}=71)$ and $2.7 \pm 3.5$ at week $54(n=50)$.

The geometric mean CRP concentration was $19.0 \mathrm{mg} / \mathrm{l}$ (95\% CI 14.2 to 25.3 ) at baseline, $6.8 \mathrm{mg} / \mathrm{l}$ (95\% CI 5.0 to 9.5$)$ at week $10(\mathrm{n}=76), 10.2 \mathrm{mg} / \mathrm{l}(95 \%$ CI 7.1 to 14.9$)$ at week 52 $(\mathrm{n}=51)$ and $11.0 \mathrm{mg} / \mathrm{l}(95 \% \mathrm{CI} 8.1$ to 15.1$)$ at last visit/withdrawal $(n=89)$. The correlation coefficients between CDEIS score and CRP level were 0.33 at week $0(n=88), 0.53$ at week 10 $(n=76)$ and 0.41 at week $54(n=51)$

\section{Central endoscopic readings}

Intraclass correlation of CDEIS scores from paired experts based on the central readings was estimated as 0.60 (95\% CI 0.39 to $0.75)$ at week $0(n=49), 0.74(95 \%$ CI 0.59 to 0.84$)$ at week 10 $(n=51)$ and $0.81(95 \%$ CI 0.65 to 0.90$)$ at week $54(n=33)$.

Table 2 CDEIS score, endoscopic response, endoscopic remission, complete endoscopic remission and mucosal healing by visit (local results only)

\begin{tabular}{|c|c|c|c|c|c|c|c|}
\hline Population & $\mathbf{N}$ & Week & $\begin{array}{l}\text { Mean } \pm \text { SD } \\
\text { CDEIS score }\end{array}$ & $\begin{array}{l}\text { Response, } \\
\%(\mathbf{9 5 \%} \mathbf{C I})\end{array}$ & $\begin{array}{l}\text { Remission, } \\
\% \text { (95\% CI) }\end{array}$ & $\begin{array}{l}\text { Complete remission, } \\
\%(95 \% \mathrm{CI})\end{array}$ & $\begin{array}{l}\text { Mucosal healing, } \\
\%(95 \% \mathrm{CI})\end{array}$ \\
\hline \multirow[t]{2}{*}{ Intention-to-treat } & 89 & 0 & $14.5 \pm 5.3$ & & & & \\
\hline & & 10 & $8.8 \pm 6.1$ & 54 (43 to 65$)$ & 37 (27 to 48 ) & 10 (5 to 18$)$ & $4(1$ to 11$)$ \\
\hline \multirow{3}{*}{$\begin{array}{l}\text { Subpopulation with } \\
\text { endoscopic assessment } \\
\text { at time point }\end{array}$} & 78 & 0 & $14.7 \pm 5.3$ & & & & \\
\hline & & 10 & $8.3 \pm 6.0$ & 62 (50 to 72 ) & 42 (31 to 54$)$ & 12 (5 to 21$)$ & 5 (1 to 13$)$ \\
\hline & 53 & 0 & $15.1 \pm 5.3$ & & & & \\
\hline
\end{tabular}

Response defined as decrease in CDEIS score of $\geq 5$, remission defined as CDEIS score $<6$, complete remission defined as CDEIS score $<3$, mucosal healing defined as no ulcers. CDEIS, Crohn's Disease Endoscopic Index of Severity. 


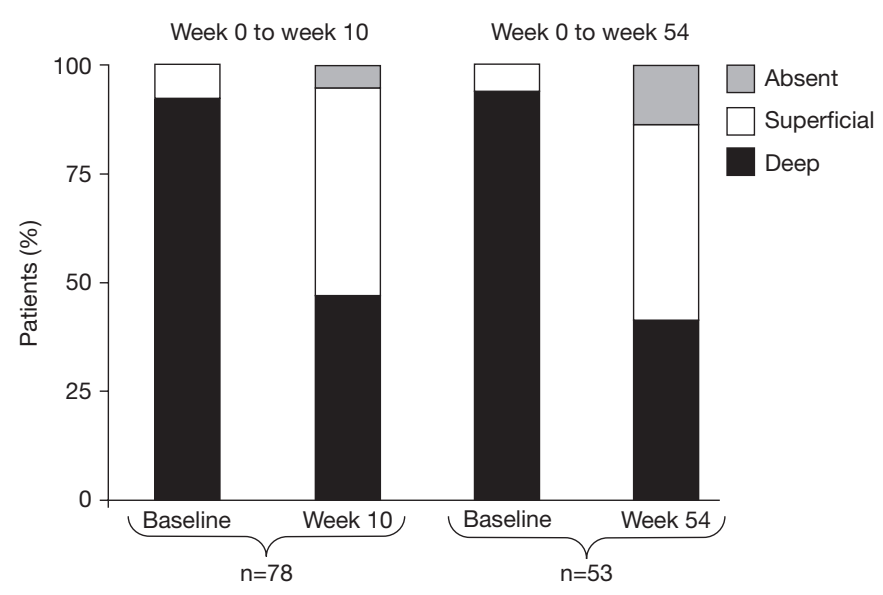

Figure 3 Ulceration classification in patients with endoscopic evaluation at baseline/week 10 and baseline/week 54 .

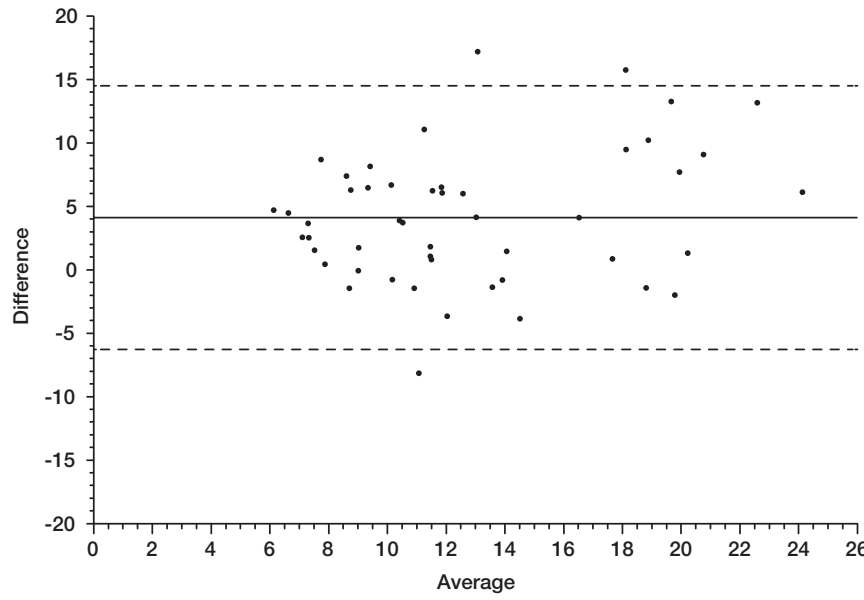

Figure 4 Agreement between local and central Crohn's Disease Endoscopic Index of Severity scores at week 0 (baseline) by the Bland-Altman method. The difference between local and central scores (positive values indicate a higher local score) as a function of their average value. The solid line represents the mean difference and the dashed lines represent the $95 \%$ agreement limits.

Table 3 Comparison of central and local readings in patients with both evaluations at baseline/week 10 and at baseline/week 54

\begin{tabular}{|c|c|c|c|c|}
\hline & \multicolumn{2}{|c|}{$\begin{array}{l}\text { Patients with both central and local readings at } \\
\text { baseline and week } 10(n=44)\end{array}$} & \multicolumn{2}{|c|}{$\begin{array}{l}\text { Patients with both central and local readings at } \\
\text { baseline and week } 54(n=28)\end{array}$} \\
\hline Baseline CDEIS: mean $\pm S D$ & $10.9 \pm 4.5$ & $14.7 \pm 5.9$ & $10.8 \pm 5.2$ & $15.1 \pm 5.9$ \\
\hline CDEIS decrease: mean $(95 \% \mathrm{CI})$ & $3.8(2.5$ to 5.1$)$ & $6.2(4.5$ to 8.5$)$ & $3.0(0.2$ to 5.7$)$ & $5.7(2.8$ to 8.6$)$ \\
\hline $\begin{array}{l}\text { Endoscopic response: } \\
\% \text { of patients }(95 \% \mathrm{Cl})\end{array}$ & 39 (24 to 55$)$ & 59 (43 to 74$)$ & 39 (22 to 59$)$ & 61 (41 to 79$)$ \\
\hline $\begin{array}{l}\text { Complete endoscopic remission: } \\
\% \text { of patients }(95 \% \mathrm{Cl})\end{array}$ & $21(10$ to 35$)$ & 14 (5 to 27$)$ & 18 (6 to 37$)$ & 21 (8 to 41 ) \\
\hline
\end{tabular}

CDEIS, Crohn's Disease Endoscopic Index of Severity.

Agreement between local and central CDEIS scores at week 0 is shown in figure 4 using the Bland and Altman method. The mean difference between local and central CDEIS scores was 4.1 with $95 \%$ limits of agreement between -6.3 and $14.5(n=49)$. Interestingly, this mean difference appeared to be higher for a high average CDEIS score. Similar results were observed at week $10(n=51)$ and week $54(n=33)$, but the mean difference was smaller (1.0 in each case, data not shown).

Seventy-eight patients had evaluable recorded endoscopies at baseline and week 10. Consent to use these recordings could not be obtained in 31 cases and recordings were inadequate in three cases, so there were 44 central readings. At week 10 the mean

Table 4 Summary of adverse events with onset during the treatment period to week 54 (intention-to-treat population)

\begin{tabular}{ll}
\hline & Patients, n (\%) \\
\hline Any adverse event & $87(98)$ \\
Adverse events leading to permanent discontinuation & $17(19)$ \\
Drug-related adverse events & $56(63)$ \\
Serious adverse events & $34(38)$ \\
Drug-related serious adverse events & $15(17)$ \\
Serious infections & $16(18)$ \\
Colon cancer & $1(1)$ \\
Death & 0 \\
\hline
\end{tabular}

CDEIS score decreased by 3.8 (95\% CI 2.5 to $5.1, \mathrm{p}<0.0001$ ) according to central reading and by 6.2 (95\% CI 4.5 to 7.8 , $\mathrm{p}<0.0001$ ) according to local reading in these 44 patients (table 3). Rates of endoscopic response, remission and complete remission assessed by central reading and by local reading in these 44 patients are shown in table 3 .

A total of 28 patients had evaluable recorded endoscopies at baseline and week 54 . The CDEIS score decreased by a mean of $3.0(95 \%$ CI 0.2 to $5.7, \mathrm{p}<0.05)$ according to central reading and by 5.7 (95\% CI 2.8 to $8.6, \mathrm{p}<0.001)$ according to local reading in these patients. Rates of endoscopic response, remission and complete remission assessed by central reading and by local reading in these 28 patients are shown in table 3.

Maintenance of response was evaluated by assessing the mean change in the CDEIS score between weeks 10 and 54. A total of 31 patients had evaluable recorded endoscopies at weeks 10 and 54. In this population the local readings were $7.7 \pm 6.4$ at week 10 and $8.3 \pm 5.9$ at week 54 (mean change $0.6,95 \%$ CI 1.3 to 2.6, $\mathrm{p}=0.50$ ); the central readings were $7.1 \pm 4.6$ at week 10 and $7.7 \pm 5.6$ at week 54 (mean change $0.5,95 \%$ CI -1.2 to 2.3, $\mathrm{p}=0.53)$.

\section{Safety}

A summary of adverse events with onset during the treatment period through week 54 is shown in table 4 . No deaths were 
observed. Colon cancer was suspected in one patient following histology results 8 days after the first endoscopy and first CZP injection. Hemicolectomy confirmed colon cancer and the study drug was permanently discontinued. The event was judged as not being related to the study medication. This was the only confirmed case of cancer; no other cases were suspected or reported. Sixteen patients had severe infection of which half were anal or perianal abscesses. No cases of tuberculosis were reported.

\section{DISCUSSION}

The MUSIC study is an open-label study specifically designed to evaluate the effect of CZP, a PEGylated anti-TNF, on endoscopic lesions in patients with active CD. It showed that endoscopic lesions were improved after treatment with CZP in patients with active severe endoscopic CD. In both the ITT population and the population with endoscopic assessment at week 10, the mean CDEIS score decreased by about $40 \%$ from baseline to week 10 (the primary outcome measure). At week 10 more than $50 \%$ of patients had an endoscopic response and more than $35 \%$ of patients had endoscopic remission. In addition, by week 10 the percentage of patients with deep ulcerations decreased by half, from $92 \%$ at baseline to $46 \%$.

The results at 1 year (54 weeks) also demonstrated a clinically meaningful reduction in mean CDEIS scores as well as rates of response, remission and complete remission similar to those seen at week 10. Maintenance of improvement between weeks 10 and 54 , based on individual patient data, was found in about $70 \%$ of those who responded and those with complete remission and over $40 \%$ of those with remission. Moreover, at weeks 10 and 54 approximately half the patients who had an endoscopic evaluation demonstrated categorical improvement in ulcerations and, by week 54 , in seven (13\%) patients no ulcerations were observed. In addition, the CDEIS score showed no significant change in the ITT population from week 10 to week 54, although it did increase slightly in those with an endoscopic evaluation at both time points.

The results of the blinded central readings of recorded endoscopies demonstrated findings similar to the local readings at weeks 10 and 54, providing confirmation of endoscopic improvement. The major difference was that the CDEIS scores were lower at all time points compared with the local readings, particularly at baseline. This difference is reflected in the lower response rates-based on an absolute decrease in CDEIS scores from baseline-in the central readings compared with the local readings. However, remission and complete remission rates were equivalent, as was the significance of the mean change in CDEIS score from baseline to week 10 .

Despite the endoscopic improvement observed, the rate of complete mucosal healing was relatively low. This may have been a consequence, at least in part, of the severity of intestinal lesions at baseline-more than $90 \%$ of patients having deep ulcerations-and the inclusion criterion for ulcerations in at least two intestinal segments. In such patients the definition of mucosal healing as the total disappearance of all mucosal ulcerations is very stringent. For example, if treatment of a patient with numerous deep mucosal ulcerations results in healing of all ulcerations except one superficial ulcer, this patient will be classified as not having achieved mucosal healing. ${ }^{5}$ In the present study we used the definitions of response and remission based on the CDEIS, ${ }^{15}$ which has been validated as a reproducible index and is also used for assessing mucosal healing in CD. ${ }^{20}$

At baseline, the severity of $\mathrm{CD}$ was evaluated based on both the CDAI (range 220-<450) and on endoscopic criteria (ulcerations in at least two intestinal segments with a CDEIS score of $\geq 8$ points). It is interesting to note that, among the 118 patients initially screened, 16 were not included because they lacked the endoscopic criteria despite clinical activity based on the CDAI. In addition, there was a weak correlation between CDAI scores and CDEIS scores at each time point of the study. This discrepancy between clinical and endoscopic activity has been observed in other studies. ${ }^{21} 22$

The value of the CDEIS as a measure of mucosal pathology depends on its reliability, which we examined in our supplementary analyses. The intraclass correlation between CDEIS scores reported by the paired expert readers was good, providing fresh evidence of the reliability of the instrument when used by experienced readers. The Bland-Altman analysis of the agreement of CDEIS scores between local and central readers showed a tendency for less expert local readers to report higher CDEIS scores than expert readers, particularly when average CDEIS scores were in the upper end of the range. This may have been due to a perceptual bias occurring during the local endoscopic reading itself, in that an observation of extensive inflammation may have led to overscoring. Taken together, these findings stress the need for training before using CDEIS.

It is important to consider our findings in light of previous research on the impact of anti-TNF therapies on endoscopic lesions and mucosal healing, recognising that comparisons are difficult because of differences in inclusion criteria, study design and definition of mucosal healing. In the ACCENT 1 study, ${ }^{23}$ an endoscopic sub-study examining mucosal healing was performed in 99 patients. $^{24}$ The CDEIS was not used in this study as an assessment of mucosal healing. At week 10, induction therapy with infliximab resulted in mucosal healing in $29 \%$ of patients (13/45) compared with $3 \%$ of patients $(1 / 29$ $p=0.006)$ who received only one infusion at baseline. Systematic maintenance therapy with infliximab 5 or $10 \mathrm{mg} / \mathrm{kg}$ every 8 weeks resulted in mucosal healing at week 54 in $44 \%$ of patients $(16 / 36)$ compared with $18 \%$ of patients $(4 / 22, p=0.041)$ who were treated with infliximab infusions episodically. Among the 99 patients enrolled in this study, 74 and 58 patients underwent follow-up endoscopic examination at weeks 10 and 54 , respectively. Mucosal healing rates reported at week 26 in the SONIC trial were $17 \%$ for the azathioprine group, $30 \%$ for the infliximab monotherapy group and $44 \%$ in the azathioprine + infliximab group. ${ }^{22}$ Again, comparisons with the SONIC trial are difficult as patients in this study were naive to both immunosuppressants and anti-TNF therapy and had shorter durations of CD (mean 2 years). The EXTEND trial ${ }^{25}$ was a placebocontrolled endoscopy trial that evaluated the effects of adalimumab on mucosal healing in 129 patients with CD involving the colon. The primary endpoint was the absence of mucosal ulceration at week 12 of treatment (endoscopic remission was a secondary outcome measure but was defined as a CDEIS score $\leq 4$, which does not correspond to the definition of remission or complete remission used in this study). Induction therapy with adalimumab $160 \mathrm{mg}$ at week 0 and $80 \mathrm{mg}$ at week 2 followed by scheduled maintenance therapy (adalimumab $40 \mathrm{mg}$ every other week) resulted in mucosal healing in $27 \%$ and $24 \%$ of patients at weeks 12 and 52, respectively, compared with 13\% and $0 \%$ of patients receiving only induction treatment with adalimumab followed by placebo ( $p=0.056$ and $p<0.001$, respectively).

The main limitation of the MUSIC study was the lack of a control group. However, spontaneous endoscopic improvement in such severe patients is very unlikely. The results were also reinforced by the blinded histological findings and blinded reading of recorded endoscopies. In both the ileum and colon, histological 
scores improved between baseline and week 10 with a similar improvement at week 54 . Independent reading of recorded paired endoscopies confirmed improvement in CDEIS scores at week 10 in the subgroup of 44 patients with active CD treated with CZP with evaluable recorded endoscopies at baseline and week 10 .

In conclusion, the results of this study show a rapid improvement of endoscopic lesions in approximately half of CZP-treated patients with moderate to severe ileocolonic CD with endoscopic lesions. Together with the established efficacy in sustaining clinical remission, ${ }^{12-14}$ this study augments the available evidence that CZP $400 \mathrm{mg}$ is effective in the treatment of patients with CD.

\author{
Author affiliations \\ ${ }^{1}$ CHU Nice, INSERM ERI-21, EA4319, University of Nice Sophia-Antipolis, Nice, France \\ ${ }^{2}$ Groupe d'Etude Thérapeutique des Affections Inflammatoires du Tube Digestif \\ (GETAID) \\ ${ }^{3}$ Hôpital Saint Louis, Paris, France \\ ${ }^{4}$ Hôpital Beaujon, Clichy, France \\ ${ }^{5}$ UCL Saint Luc, Brussels, Belgium \\ ${ }^{6} \mathrm{CHU}$ Amiens, Amiens, France \\ ${ }^{7}$ Special Medical Practice, Berlin, Germany \\ ${ }^{8}$ Academic Medical Centre, Amsterdam, The Netherlands and Imelda General \\ Hospital, Bonheiden, Belgium \\ ${ }^{9}$ UCB Pharma, Braine L'Alleud, Belgium \\ ${ }^{10}$ University Hospital Gasthuisberg, Leuven, Belgium \\ ${ }^{11} \mathrm{CHU}$ Reims, Reims, France \\ ${ }^{12}$ Antwerp University Hospital, Antwerp, Belgium \\ ${ }^{13}$ INSERM U717, Université Paris 7, Paris, France \\ ${ }^{14}$ Hôpital Lariboisière, Université Paris 7, Paris, France \\ ${ }^{15} \mathrm{CHU}$ Lille, INSERM-CIC9301 and Universite Lille Nord de France, Lille, France
}

\begin{abstract}
Acknowledgements The authors thank Edouard Louis and Matthieu Allez for providing expert central readings of recorded endoscopies and Alexandre Auborg (CHRU Tours), Jessica Coelho (APHP Hôpital Lariboisière), Clotilde Baudry (APHP Hôpital Saint Louis) and Stéphanie Viennot (CHU Caen) for their participation in the central readings process. Editorial assistance in the preparation of this article was provided by B Kadish of PPSI (a PAREXEL company) and was funded by UCB Pharma.
\end{abstract}

Contributors The study protocol was developed collaboratively by the authors and the clinical development team at UCB. XH, ML and JFC led the development of each draft; all other authors critically reviewed each draft and contributed to the revision process. Data analysis was conducted at UCB under the direction of EE according to a statistical analysis plan; these analyses were critically reviewed by JYM, XH and JFC who directed the execution of additional analyses. All authors approved the final version of the manuscript. Assistance provided by the medical writer included: working with the lead authors to develop drafts, incorporating input from coauthors, managing the administrative aspects of the process and overseeing the copyediting, formatting and styling of the manuscript, figures and tables.

Funding The MUSIC study (NCT00297648) was funded by UCB Pharma.

Competing interests $\mathrm{XH}$ received funding from UCB Pharma, Baxter, Fresenius Kabi, Vifor for advisory activity, as a member on an advisory board and from Abbott, Nestlé, Norgine, Nutricia and Schering-Plough for educational activities. ML served as a consultant for UCB Pharma and participated in continuing medical education events supported by unrestricted educational grants from UCB Pharma. He died on 26 August 2010. YB has been reimbursed by Schering-Plough and Abbott for attending several conferences and has been paid by Schering-Plough, Abbott, Ferring, Norgine, HAC Pharma, BMS and Teva for running educational programmes. OD has received fees from MSD, Abbott and Ferring for speaking and from MSD and Abbott for educational activities. J-LD has no disclosure information or competing interests to report. MM has received payment for conducting clinical studies for Roche Pharma, MSD Pharma, Ferring, Merckle-Recordati, Shire, Merz Pharma, Viofor Pharma, Abbott, UCB Pharma, Dr. Falk Pharma and AstraZeneca and has been reimbursed for attending conferences by several of these companies. GD'H has received consulting fees from UCB Pharma and has been paid to serve on Speakers Bureau/Advisory Committees for UCB Pharma. KM was an employee of UCB Pharma during development of this manuscript. EE is an employee of UCB Pharma. SV has received fees from UCB, Abbott, Centocor and MSD for running educational programmes and has a research chair from UCB. HB-B has been reimbursed by Abbott, Schering-Plough and UCB for attending several conferences and has received fees from Abbott and Schering-Plough for speaking. TM received lecture fees, grant support and research support from UCB Pharma. PM has served as a consultant for UCB Pharma and has participated in continuing medical education events supported by unrestricted educational grants from UCB Pharma.
J-YM has no competing interests to report. J-FC has received consulting fees from Abbott Laboratories, ActoGeniX, Albireo Pharma, Amgen, AstraZeneca, Bayer AG, Biogen Idec, Boehringer Ingelheim GmbH, Bristol-Myers Squibb, Cellerix, Centocor, ChemoCentryx, Cosmo Technologies, Danone Research, Elan Pharmaceuticals, Genentech, Giuliani SpA, Given Imaging, Glaxo Smith Kline, Hutchison MediPharma, MSD, Millennium Pharmaceuticals (now Takeda), Neovacs, Ocera Therapeutics, Pfizer Shire Pharmaceuticals, Prometheus Laboratories, Sanofi-Aventis, Schering-Plough, Synta Pharmaceuticals, Teva, Therakos, UCB Pharma and Wyeth; has served on advisory committees for Abbott Laboratories, Centocor, Danone, Elan, MSD, Millennium Pharmaceuticals (now Takeda), Schering-Plough and UCB Pharma; has received speaking fees from Abbott Laboratories, Centocor, Elan Pharmaceuticals, Given Imaging, MSD, Otsuka America Pharmaceutical, Schering-Plough, Shire

Pharmaceuticals, Tillotts Pharma and UCB Pharma; has received grant support from Abbott Laboratories, Astra-Zeneca, Ferring, MSD, Schering-Plough and UCB Pharma; and has stock ownership for Intestinal Biotech Development, Lille, France.

Ethics approval The protocol, all protocol amendments, and the informed consent document were reviewed and approved by an independent ethics committee or institutional review board at each site. All patients gave their informed consent to participate in the study before any study-related procedures were performed.

Provenance and peer review Not commissioned; externally peer reviewed.

Open Access This is an Open Access article distributed in accordance with the Creative Commons Attribution Non Commercial (CC BY-NC 3.0) license, which permits others to distribute, remix, adapt, build upon this work non-commercially, and license their derivative works on different terms, provided the original work is properly cited and the use is non-commercial. See: http://creativecommons.org/ licenses/by-nc/3.0/

\section{REFERENCES}

1. Podolsky DK. Inflammatory bowel disease. N Engl J Med 2002;347 417-29.

2. van Dullemen HM, van Deventer SJ, Hommes DW, et al. Treatment of Crohn's disease with anti-tumor necrosis factor chimeric monoclonal antibody (cA2). Gastroenterology 1995;109:129-35.

3. Modigliani R, Mary JY, Simon JF, et al. Clinical, biological, and endoscopic picture of attacks of Crohn's disease. Evolution on prednisolone. Groupe d'Etude Therapeutique des Affections Inflammatoires Digestives. Gastroenterology 1990;98:811-18

4. Rutgeerts $\mathbf{P}$, Vermeire $\mathrm{S}$, Van Assche G. Mucosal healing in inflammatory bowel disease: impossible ideal or therapeutic target? Gut 2007:56:453-5.

5. Pineton de Chambrun G, Peyrin-Biroulet L, Lemann M, et al. Clinical implications of mucosal healing for the management of IBD. Nat Rev Gastroenterol Hepatol 2010:7:15-29.

6. Baert F, Moortgat L, Van Assche G, et al. Mucosal healing predicts sustained clinical remission in patients with early-stage Crohn's disease. Gastroenterology 2010;138:463-8.

7. D'Haens G, Daperno M. Advances in medical therapy for Crohn's disease. Curr Gastroenterol Rep 2002;4:506-12.

8. Rutgeerts P, Diamond RH, Bala M, et al. Scheduled maintenance treatment with infliximab is superior to episodic treatment for the healing of mucosal ulceration associated with Crohn's disease. Gastrointest Endosc 2006; 63:433-42.

9. Froslie KF, Jahnsen J, Moum BA, et al. Mucosal healing in inflammatory bowel disease: results from a Norwegian population-based cohort. Gastroenterology 2007; 133:412-22.

10. Schnitzler $\mathbf{F}$, Fidder $H$, Ferrante $M$, et al. Mucosal healing predicts long-term outcome of maintenance therapy with infliximab in Crohn's disease. Inflamm Bowel Dis 2009;15:1295-301.

11. D'Haens G. Anti-TNF-alpha treatment strategies: results and clinical perspectives. Gastroenterol Clin Biol 2009;33(Suppl 3):S209-16.

12. Sandborn WJ, Feagan BG, Stoinov $S$, et al. Certolizumab pegol for the treatment of Crohn's disease. N Engl J Med 2007;357:228-38.

13. Schreiber S, Rutgeerts P, Fedorak RN, et al. A randomized, placebo-controlled trial of certolizumab pegol (CDP870) for treatment of Crohn's disease. Gastroenterology 2005; 129:807-18.

14. Schreiber S, Khaliq-Kareemi M, Lawrance IC, et al. Maintenance therapy with certolizumab pegol for Crohn's disease. N Engl J Med 2007;357:239-50.

15. Mary JY, Modigliani R. Development and validation of an endoscopic index of the severity for Crohn's disease: a prospective multicentre study. Groupe d'Etudes Therapeutiques des Affections Inflammatoires du Tube Digestif (GETAID). Gut 1989;30:983-9.

16. D'Haens GR, Geboes K, Peeters M, et al. Early lesions of recurrent Crohn's disease caused by infusion of intestinal contents in excluded ileum. Gastroenterology 1998;114:262-7

17. Mary JY, Lemann M, Colombel J-F, et al. Endoscopic remission and response in Crohn's disease: an objective definition using the CDElS. Gut 2006;37(Suppl 1): A55.

18. D'Haens G, van Deventer S, Van Hogezand R, et al. Endoscopic and histological healing with infliximab anti-tumor necrosis factor antibodies in Crohn's disease: a European multicenter trial. Gastroenterology 1999;116:1029-34. 
19. Bland JM, Altman DG. Statistical methods for assessing agreement between two methods of clinical measurement. Lancet 1986;1:307-10.

20. Sandborn WJ, Feagan BG, Hanauer SB, et al. A review of activity indices and efficacy endpoints for clinical trials of medical therapy in adults with Crohn's disease. Gastroenterology 2002;122:512-30.

21. Cellier C, Sahmoud T, Froguel E, et al. Correlations between clinica activity, endoscopic severity, and biological parameters in colonic or ileocolonic Crohn's disease. A prospective multicentre study of 121 cases. The Groupe d'Etudes Therapeutiques des Affections Inflammatoires Digestives. Gut 1994; $\mathbf{3 5}: 231-5$

22. Colombel JF, Sandborn WJ, Reinisch W, et al. Infliximab, azathioprine, or combination therapy for Crohn's disease. N Engl J Med 2010;362:1383-95.
23. Hanauer SB, Feagan BG, Lichtenstein GR, et al. Maintenance infliximab for Crohn's disease: the ACCENT I randomised trial. Lancet 2002;359:1541-9.

24. Rutgeerts P, Feagan BG, Lichtenstein GR, et al. Comparison of scheduled and episodic treatment strategies of infliximab in Crohn's disease. Gastroenterology 2004;126:402-13.

25. Rutgeerts P, D'Haens G, Van Assche G, et al. Adalimumab induces and maintains mucosal healing in patients with moderate to severe ileocolonic Crohn's disease: data from the EXTEND Trial. Gastroenterology. Published Online First: 8 February 2012,22326435 